\title{
Classifying Subgroups of Solvable Groups
}

\author{
By
}

\author{
J. F. McClendon*
}

Let $G$ and $K$ be groups (finite or infinite). Two very general questions that might reasonably be asked are (1) does $G$ contain an isomorphic copy of $K$ ? (2) can the isomorphic copies of $K$ in $G$ be classified or (in the finite case) counted? The present paper can be viewed as directed toward the second question although the first is touched on lightly. Actually it will be seen that most results concern subgroups that are isomorphic to $K$ in a certain way. Also the answer is in terms of one dimensional cohomology. So it is perhaps accurate to say that the present paper studies relationships between the subgroup structure of a group and its low dimensional cohomology.

One result which can be stated without too much terminology is the following (Corollary 3.7). Let $G=G_{0} \supset G_{1} \cup \ldots \supset G_{s}=1$ be a solvable series with $A_{i}=G_{i-1} / G_{i} .\{2, \ldots, s\} \supset I$ and $B_{i}=1, \quad i \in I, B_{i}=A_{i}, i \notin I$. Suppose that if $i \in I$ and $G / G_{i-1} \supset W$ and $W$ covers $B_{1}, \ldots, B_{i-1}$ then $H^{j}\left(W, A_{i}\right)=0, j=1,2$. Then (1) $G$ has a subgroup $K$ covering $B_{1}, \ldots, B_{s}$ and any two such are conjugate. (2) all such $\leftrightarrow \Pi_{i \in I}\left(A_{i} /\right.$ $\left.A_{i}^{K / K_{i-1}}\right)$. This generalizes the classical theorem of $P$. Hall and the exact relationship to Hall's theorem is discussed in Section 3. A more typical result is Theorem 2.4 whose conclusion reads

$$
S\left((K, \bar{K}) \subset G, E, B_{0}, M\right)=\cup H^{1}\left(J, \bar{J}, u^{*} A\right) / H^{1}\left(J, \bar{J}, u^{*} t B\right) .
$$

The left hand side is a certain set of subgroups of $G$ isomorphic to $K$.

The paper is organized as follows. In Section 1 sets of subgroups

Communicated by S. Takasu, April 23, 1974.

* Department of Mathematics, University of Kansas, Kansas 66045, U. S. A. 
of an abelian extension are studied which are defined by given maps. In Section 2 it is shown that sometimes the dependence on maps can be dropped. In Section 3 the results of Sections (1) and (2) on abelian extensions are spliced to get results on solvable groups. The results of this section are of a splitting nature. That is, sufficiently strong hypotheses are used so that one can count subgroups simply by counting the number produced at each stage and multiplying the numbers. I hope to study some non-splitting situations at another time. In an appendix some results on relative cohomology and derivations are recalled.

\section{§1. Subgroups of abelian extensions which extend given maps.}

Let $K$ be a group and $A$ a $K$-module. Recall that a derivation $\alpha: K \rightarrow A$ is a function such that $\alpha(x y)=\alpha(x)+x \alpha(y)$. Sometimes $A$ will be written multiplicatively and the condition will be written $\alpha(x y)=\alpha(x)\left(x^{*} \alpha(y)\right)$. If $\alpha$ and $\beta$ are derivations then their sum is defined by $(\alpha+\beta)(x)=\alpha(x)+\beta(x)$. It is easily checked that the set $\operatorname{Der}(K, A)$ is an abelian group under this operation. If $\bar{K}$ is a subgroup of $K$ let $\operatorname{Der}(K, \bar{K}, A)$ be the subset of $\operatorname{Der}(K, A)$ satisfying $\alpha(x)=0$ for $x \in \bar{K}$. It is a subgroup of $\operatorname{Der}(K, A)$. Let $\operatorname{In}(K, A)$ be the group of inner derivations, that is, derivations of the form $\alpha_{a}$ where $a \in A, \alpha_{a}(x)=a-x a$ and $\operatorname{In}(K, \bar{K}, A)$ the subgroup of inner derivations trivial over $\bar{K}$. Note that if $\alpha \in \operatorname{Der}(K, A)$ then $\alpha(1)=0$ so $\operatorname{Der}(K, A)=\operatorname{Der}(K, 1, A)$. If $B$ is a normal subgroup of $K$ such that $B \subset \bar{K}$ acting trivially on $A$ then $\operatorname{Der}(K, \bar{K}, A)=$ $\operatorname{Der}(K / B, \bar{K} / B, A)$. In particlar, if $\bar{K}$ is normal and acts trivially on $A$ we have $\operatorname{Der}(K, \bar{K}, A)=\operatorname{Der}(K / \bar{K}, 1, A)=\operatorname{Der}(K / \bar{K}, A)$. In this case relative derivations are more a convenience than a necessity since we can reduce to the absolute case without even changing coefficients.

On the other hand, for any subgroup $\bar{K}$, $\operatorname{Der}(K, \bar{K}, A)$ can be viewed as the one dimensional cohomology group $H^{1}(K, \bar{K} ; A)$ where the latter is defined by Takasu [7]. Namely, we set $I(K, \bar{K}, Z)=$ 


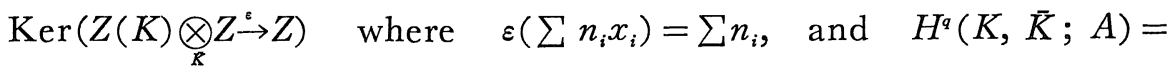
$\operatorname{Ext}^{q-1}(I(K, \bar{K}, Z), A)$ for $q \geqq 1$, and we have an exact sequence

$$
\begin{aligned}
& 0 \rightarrow A^{K} \rightarrow A^{\mathbb{R}^{\delta}} \rightarrow H^{1}(K, \bar{K} ; A) \rightarrow H^{1}(K ; A) \rightarrow H^{1}(\bar{K} ; A) \stackrel{\delta}{\rightarrow} \\
& H^{2}(K, \bar{K} ; A) \rightarrow \ldots
\end{aligned}
$$

where $H^{1}(K, \bar{K} ; A)=\operatorname{Der}(K, \bar{K}, A)$. Here we note that $H^{1}(K ; A) \simeq$ $\operatorname{Der}(K, A) / \operatorname{In}(K, A)$ which differs from $\operatorname{Der}(K, A)$ itself, and this is the point where relative cohomology is necessary to study the set of derivations. See [1] also, but note the different indexing.

In this section we will study the following situation.

$$
\begin{aligned}
& E: \quad 1 \rightarrow A \stackrel{i}{\rightarrow} G \stackrel{p}{\rightarrow} D \rightarrow 1 \\
& \underset{1 \rightarrow B \rightarrow K \rightarrow J \rightarrow 1}{\cup} \quad B \subset \bar{K} \subset K
\end{aligned}
$$

Here $E$ is an abelian group extension (the sequence is exact and $A$ is abelian). $\quad K$ is a subgroup of $G$ and $B=B(K)=K \cap A, J=J(K)=$ $p(K) . \quad \bar{K}$ is a subgroup of $K$ containing $B$ and need not be normal in $K$ and $\bar{J}=p \bar{K}$. A gets a left $J$-module structure by $(x B) a=x a x^{-1}$ for $x \in K . \quad B$ is a sub $J$-module. The map $K \rightarrow J$ gives both $K$-module structures. In particular, $A$ has a natural $D$-module structure.

1.1 Definition. (a) Suppose $K$ and $L$ are subgroups of $G$. $A$ homomorphism $\phi: K \rightarrow L$ is an $E$-homomorphism if $\phi(K \cap A) \subset L \cap A$. It is an $E$-isomorphism if it has an inverse which is also an $E$ homomorphism. It is an $E$-monomorphism if it is an $E$-isomorphism onto its image.

(b) $S(K \subset G, E)=$ all subgroups of $G$ which are $E$-isomorphic to $K$.

(c) Let $t: \bar{K} \rightarrow G, u: J \rightarrow D$ be given monomorphisms. Define $S(K \subset G, E, t / u)$ to be all $L$ in $S(K \subset G, E)$ such that there is some $E$-monomorphism $\phi: K \rightarrow G$ with $\phi=t$ on $\bar{K}, p \phi=u p$, and $\phi K=L$.

(d) Let $u: J \rightarrow D$. Then $u^{*} A$ is $A$ with the $J$-module structure from $u$. Note that if $p \phi=u p$ then in $u^{*} A$ we have $x^{*} a=\phi(x) a \phi(x)^{-1}$ : Note also that $\left(J, u^{*} A\right)$ and $(u(J), A)$ are isomorphic in the sense of change of groups.

Monomorphisms $t:(\bar{K}, B) \rightarrow(G, A), u: J \rightarrow D$ are compatible if (1) $t$ induces $u$ on $\bar{J}$ and (2) $t: B \rightarrow A$ is a homomorphism of 
$J$-modules $B \rightarrow u^{*} A$. Note that $S(K \subset G, E, t / u) \neq \phi \Rightarrow t, u$ are compatible.

Let $L, L^{\prime} \in S=S(K \subset G, E, t / u)$ and $\phi: K \rightarrow G, \phi^{\prime}: K \rightarrow G$ the $E$ monomorphisms from (c) above. Define $d=d\left(L, L^{\prime}\right): K \rightarrow G$ by $d=\phi^{\prime} \phi^{-1}$.

1.2 Lemma. $d\left(L, L^{\prime}\right) \in \operatorname{Der}\left(K, \bar{K}, u^{*} A\right)=\operatorname{Der}\left(J, \bar{J}, u^{*} A\right)$.

Proof. $p \phi=u p=p \phi^{\prime}$ so $d(K) \subset A$ and $\phi=\phi^{\prime}$ on $\bar{K}$ so $d(\bar{K})=1$. Also $d(x y)=\phi^{\prime}(x y) \phi(x y)^{-1}=\phi^{\prime}(x) d(y) \phi(x)^{-1}=\phi^{\prime}(x) \phi(x)^{-1}(\phi(x) d(y)$ $\left.\phi(x)^{-1}\right)=d(x)\left(x^{*} d(y)\right)$ so $d$ is a derivation to $u^{*} A$.

1. 3 Theorem. Suppose $L \in S=S(K \subset G, E, t / u)$. Define

$$
\Delta=\Delta_{L}: S \rightarrow \operatorname{Der}\left(J, \bar{J}, u^{*} A\right) / \operatorname{Der}\left(J, \bar{J}, u^{*} t(B)\right)
$$

by $\Delta\left(L^{\prime}\right)=d\left(L^{\prime}, L\right)$. Then $\Delta$ is a bijection.

Proof. Let $M$ be the set of all $E$-monomorphisms $\phi: K \rightarrow G$ such that $p \phi=u p$. Define an action of $\operatorname{Der}\left(K, \bar{K}, u^{*} A\right)$ on $M$ by $(\alpha \phi)(x)=\alpha(x) \phi(x)$. Let $\alpha \phi=\phi^{\prime}$. Then $\phi^{\prime}$ is a homomorphism since $\phi^{\prime}(x y)=\alpha \phi(x y)=\alpha(x)\left(x^{*} \alpha(y)\right) \phi(x) \phi(y)=\alpha(x) \phi(x) \alpha(y) \phi(x)^{-1} \phi(x)$ $\phi(y)=\phi^{\prime}(x) \phi^{\prime}(y)$. Now $x \in B \Rightarrow \alpha(x)=1$ so $\phi^{\prime}=\phi$ on $B$ and so $\phi^{\prime}$ monic on $B$ and gives a homomorphism $\bar{\phi}: J \rightarrow D$. It is easily checked that $\bar{\phi}=u$ and so $\bar{\phi}$ is monic. Now let $M \supset N=$ those momomorphisms which restrict to $t$ on $\bar{K}$. $\operatorname{Der}\left(K, \bar{K}, u^{*} A\right)$ acts on $N$ since $\phi^{\prime}=\phi$ on $\bar{K}$. The action is transitive since if $\dot{\phi}_{1}, \phi_{2} \in N$ then $d\left(\phi_{2}, \phi_{1}\right) \phi_{1}=\phi_{2}$ and by the lemma $d$ is in $\operatorname{Der}\left(K, \bar{K}, u^{*} A\right)$. The action is clearly faithful.

There is a surjection $N \rightarrow S$, namely, $\phi \rightarrow \phi(K) \subset G$. In order to prove the theorem we must show exactly that:

$$
\alpha \phi(K)=\alpha^{\prime} \phi(K) \Leftrightarrow \alpha^{\prime} \alpha^{-1} \in \operatorname{Der}\left(K, \bar{K}, u^{*} t(B)\right)
$$

for some (and hence any) $\phi \in N$.

First let $\beta=\alpha^{\prime} \alpha^{-1} \in \operatorname{Der}\left(K, \bar{K}, u^{*} t(B)\right)$. Then $\alpha \phi(x)=\beta(x)^{-1}$ $\alpha^{\prime}(x) \phi(x) \in t(B)\left(\alpha^{\prime} \phi\right)(K)$. But $\alpha^{\prime} \phi|B=\phi| B=t \mid B$ so $t(B) \subset \alpha^{\prime} \phi(B)$ 
and hence $\alpha \phi(K) \subset \alpha^{\prime} \phi(K)$ and vice-versa, proving half the claim.

Now suppose $\alpha \phi(K)=\alpha^{\prime} \phi(K)$. Then $\alpha^{\prime} \phi=(\alpha \phi) \circ \nu$ where $\nu: K \rightarrow K$ is an $E$-automorphism of $K$ giving the identity on $\bar{K}$ and $J$. Now define $\mu(x)=\nu(x) x^{-1}$ and check that $\mu \in \operatorname{Der}(K, \bar{K}, B)$. [Check that $\nu(K), 1_{K}(K) \in S\left(K \subset G, E, 1_{R} / 1_{J} K\right)$ and the lemma gives $\mu=$ $d(\nu, 1) \in \operatorname{Der}(K, \bar{K}, B)]$. Note that $(\alpha \phi) \circ \nu=(\phi \circ \mu) \alpha \phi$. The reason is that $(\alpha \phi)(\nu(x))=\alpha(\mu(x) x) \phi(\mu(x) x)=\alpha(\mu(x)) \phi(\mu(x)) \alpha(x) \phi(\mu(x))^{-1}$ $\phi(\mu(x)) \phi(x)=\phi(\mu(x)) \alpha(x) \phi(x)$ [since $\mu(x) \in B \Rightarrow \alpha \mu(x)=1]=$ $\{((\phi \circ \mu) \alpha) \phi\}(x)$. We see that $\alpha^{\prime} \phi=(\alpha \phi) \circ \nu=(\phi \circ \mu) \alpha \phi$. Check that $\phi \circ \mu \in \operatorname{Der}\left(K, \bar{K}, u^{*} t(B)\right)$ and $\alpha^{\prime} \alpha^{\prime-1}=\phi \mu$. This proves the theorem.

Note that it follows from the theorem that $d\left(L^{\prime}, L\right)$ is a well defined element of $\operatorname{Der}\left(J, \bar{J}, u^{*} A\right) / \operatorname{Der}\left(J, \bar{J}, u^{*} t B\right)$ and depends only on $L$ and $L^{\prime}$. Also $d\left(L, L^{\prime}\right)=0 \Leftrightarrow L=L^{\prime}$.

1. 4 Corollary. Suppose $\bar{K}$ normal in $K$ and $L \in S=S(K \subset G, E$, $t / u)$. Then

$$
S \leftrightarrow \operatorname{Der}\left(J / \bar{J}, u^{*} A^{R}\right) / \operatorname{Der}\left(J / \bar{J}, u^{*} t B^{R}\right)
$$

Derivations can always be viewed as absolute one dimensional cocycles, and they can also be viewed as one dimensional relative cohomology classes and this leads to the following corollary.

1.5 Corollary. 1) $\Delta_{L}: S \rightarrow H^{1}\left(J, \bar{J}, u^{*} A\right) / H^{1}\left(J, \bar{J}, u^{*} t B\right)=$ $H^{1}\left(K, \bar{K}, u^{*} A\right) / H^{1}\left(K, \bar{K}, u^{*} t B\right)$ is a bijection.

2) If $\bar{K}$ is normal in $K$ then

$\Delta_{L}: S \rightarrow H^{1}\left(J / \bar{J}, 1, u^{*} A^{R}\right) / H^{1}\left(J / \bar{J}, 1, u^{*} t B^{R}\right)$ is a bijection.

Notice that if we only wanted part 2 we could have used absolute derivations only in Theorem 1.3. However, even in this case relative derivations seem more natural and are required for the cohomology statement anyway-since $(J / \bar{J}, 1)$ can't be changed to $J / \bar{J}$ without changing coefficients. The following simple (simplest) example shows this. Let $G=S_{3}=$ symmetric group on 3 symbols and let $A \rightarrow G \rightarrow D$ be $A \rightarrow S_{3} \rightarrow C_{2}$ where $A$ is the cyclic subgroup of order 3 and in 
general $C_{n}$ is the cyclic group with $n$ elements. Let $B \rightarrow K \rightarrow J$ be $1 \rightarrow K \rightarrow K$ where $K$ is the subgroup $\{1,(12)\}$ of order 2. Let $\bar{K}=$ $B=1$ and $u: J \rightarrow D$ be the isomorphism. Then we have $H^{1}\left(K / \bar{K}, 1, u^{*} A\right) /$ $H^{1}\left(K / \bar{K}, 1, u^{*} t B\right)=H^{1}\left(C_{2}, 1, C_{3}\right)=C_{3}$. It is easily seen directly that $S(K \subset G, E, t / u)$ does indeed have three elements. However, $H\left(K / \bar{K}, u^{*} A\right) / H^{1}\left(K / \bar{K}, u^{*} t(1)\right)=H^{1}\left(C_{2} ; C_{3}\right)=\{0\}$.

1.6 Corollary. $S \leftrightarrow \operatorname{Ker} \delta: H^{1}\left(J, \bar{J}, u^{*}(A / t B)\right) \rightarrow H^{2}\left(J, \bar{J}, u^{*} t B\right)$

Proof. $0 \rightarrow B \rightarrow A \rightarrow A / B \rightarrow 0$ gives an exact sequence

$$
0 \rightarrow H^{1}(J, \bar{J}, B) \rightarrow H^{1}(J, \bar{J}, A) \rightarrow H^{1}(J, \bar{J}, A / B) \rightarrow H^{2}(J, \bar{J}, B) \rightarrow \ldots
$$

(where $A$ is $u^{*} A$ etc.) and the corollary follows.

1. 7 Corollary. Assume $\operatorname{Ker} \delta: H^{1}\left(\bar{J}, u^{*} t B\right) \rightarrow H^{2}\left(J, \bar{J}, u^{*} t B\right)$ is 0 and $H^{1}\left(J, u^{*} t B\right) \rightarrow H^{1}\left(J, u^{*} A\right)$ is isomorphic. Then

$$
S \leftrightarrow u^{*} A^{J} /\left[u^{*} A^{J}+u^{*} t B^{J}\right]
$$

Proof. The hypotheses and the pair $(J, \bar{J})$ give the following commutative diagram with exact rows. Here $A$ is $u^{*} A$ and $B$ is $u^{*} t B$.

$$
\begin{aligned}
& 0 \rightarrow A^{J} \rightarrow A^{J} \rightarrow H^{1}(J, \bar{J}, A) \rightarrow H^{1}(J, A) \\
& \stackrel{\uparrow}{\uparrow} \underset{\uparrow}{\cong} \\
& 0 \rightarrow B^{J} \rightarrow B^{J} \rightarrow H^{1}(J, \overline{\bar{J}}, B) \rightarrow H^{1}(J, B) \rightarrow 0
\end{aligned}
$$

The result now follows from a diagram argument.

1.8 Corollary. (1) Suppose $\bar{K}=B$ and $H^{1}\left(J ; u^{*} t B\right) \rightarrow H^{1}\left(J ; u^{*} A\right)$ isomophic. Then

$$
S \leftrightarrow u^{*} A /\left[u^{*} A^{J}+u^{*} t B\right]
$$

(2) Suppose $\bar{K}=B=1$ and $H^{1}\left(J ; u^{*} A\right)=0$. Then $S \leftrightarrow u^{*} A / u^{*} A^{K}$.

Proof. Since $\bar{J}=1$ these results follow from corollary 1.7 .

Note that the $S_{3}$ example mentioned above illustrates part (2) 
since $H^{1}\left(C_{2} ; u^{*} C_{3}\right)=0$ and $S \leftrightarrow A / A^{K}=A=C_{3}$.

In general a conjugate of an element of $S(K \subset G, E)$ is another element of $S(K \subset G, E)$.

Let $A^{\prime}=A \cap$ centralizer of $t \bar{K}$. Then $S(K \subset G, E, t / u)$ is closed under conjugation by elements of $A^{\prime}$.

1.9 Theorem. Let $L \in S(K \subset G, E, t / u)$ and $\Delta=\Delta_{L}$. Then

$$
S \leftarrow \Delta \longrightarrow \operatorname{Der}\left(J, \bar{J}, u^{*} A\right) / \operatorname{Der}\left(J, \bar{J}, u^{*} t B\right)
$$

$A^{\prime}$-conjugates of $L \longleftrightarrow \operatorname{In}\left(J, \bar{J}, u^{*} A\right) / \operatorname{In}\left(J, \bar{J}, u^{*} A\right) \cap \operatorname{Der}\left(J, \bar{J}, u^{*} B\right)$

Proof. Let $\Gamma=\Delta^{-1}$. It will suffice to show that $\Gamma\langle\alpha\rangle$ is conjugate to $L$ iff $\alpha^{\prime} \in \operatorname{In}\left(J, \bar{J}, u^{*} A\right)$ for some $\alpha^{\prime} \in\langle\alpha\rangle$. Let $L=\phi(K)$ where $\phi \in N$ as in the proof of Theorem 1.3. First suppose $\alpha=$ $\alpha^{a} \in \operatorname{In}\left(J, \quad \bar{J}, \quad u^{*} A\right) . \quad$ Then $\quad(\alpha \phi)(x)=\alpha(x) \phi(x)=a\left(x^{*} a\right)^{-1} \phi(x)=$ $a\left[\phi(x) a \phi(x)^{-1}\right]^{-1} \phi(x)=a \phi(x) a^{-1} \phi(x)^{-1} \phi(x)=a \phi(x) a^{-1}$, so $\left(\alpha_{a} \phi\right)(K)=$ $c_{a}(\phi(K))$ and $\Gamma\langle\alpha\rangle$ is an $A$-conjugate of $L$. Conversely, $\Gamma\langle\alpha\rangle=L^{\prime}=$ $a L a^{-1}$ implies $\alpha \phi(K)=\left(c_{a} \circ \phi\right)(K)=\left(\alpha_{a} \phi\right)(K)$ and so $\langle\alpha\rangle=\left\langle\alpha_{a}\right\rangle$.

1.10 Corollary. Assume $H^{1}\left(J, \bar{J}, u^{*} A\right)=\delta u^{*} A^{J}+i_{*} H^{1}\left(J, \bar{J}, u^{*} t B\right)$. Then all elements of $S(K \subset G, E, t / u)$ are conjugate by elements of $A^{\prime}$.

Proof. Here, $u^{*} A^{J}=H^{\circ}\left(\bar{J} ; u^{*} A\right) \stackrel{\delta}{\rightarrow} H^{1}\left(J, \bar{J} ; u^{*} A\right)$ is part of the exact sequence of $(J, \bar{J})$. The corollary follows from 1.9 since $\operatorname{In}(J, \bar{J}, A) /[\operatorname{In}(J, \bar{J}, A) \cap \operatorname{Der}(J, \bar{J}, B)]=[\operatorname{In}(J, \bar{J}, A)+\operatorname{Der}(J, \bar{J}, B)] /$ $\operatorname{Der}(J, \bar{J}, B)$ and by the assumptions that the last is $\operatorname{Der}(J, \bar{J}, A) /$ $\operatorname{Der}(J, \bar{J}, B)=S$.

\section{§2. Subgroups of abelian extensions which extend given subgroups}

The notation of Section 1 will be used here.

2.1 Definition. Let $G \supset M \supset B_{0}, \mathrm{D} \supset J_{0}$. Define $S((K, \bar{K}) \subset G$, $\left.E, B_{0} / J_{0}, M\right)$ to be all $L$ in $S(K \subset G, E)$ such that there is an 
$E$-monomorphism $\phi: K \rightarrow G$ with $\phi B=B_{0}, \phi(K)=L \phi J=J_{0}, \phi \bar{K}=M$. Define $\mathrm{S}\left((K, \bar{K}) \subset G, E, B_{0}, M\right)$ by requiring only $\phi B=B_{0}$ and $\phi(\bar{K})=M$. If $B=\bar{K}$ drop $M$ and $\bar{K}$ from the notation.

Suppose $t: \bar{K} \rightarrow G, \mu: J \rightarrow D$ are given monomorphisms with $t(\bar{K})=M$, $t(B)=B_{0}, \mu(J)=J_{0}$. Then we have

$$
S=S(K \subset G, E, t / \mu) \subset S^{\prime}=S\left((K, \bar{K}) \subset G, E, B_{0} / J_{0}, M\right)
$$

2.2 Definition. $(K, \bar{K}, B)$ is a $(t / u)$-triple if $S=S^{\prime}$.

We note here the following basic examples of $(t / u)$-triples.

(1) $J=1$. Then $(B, B, B)$ is a $(t / 1)$-triple.

(2) $S$ non-empty, $B=A$. Then $(K, A, A)$ is a (id/u)-triple.

(3) $B=1$. Then $(K, 1,1)$ is a $(1 / u)$-triple.

(1) and (2) are clear since in each case $S$ and $S^{\prime}$ have exactly one element. (3) can be checked directly without much trouble, however, it follows from the theorem below.

Let $\operatorname{Aut}(K, \bar{K}, B)$ be the group of automorphisms of $K$ sending $\bar{K}$ to itself and $B$ to itself (similarly for $\operatorname{Aut}(K, \bar{K})$ etc.). Let $\delta \in \operatorname{Aut}(\bar{K}, B)$ and $\varepsilon \in \operatorname{Aut}(J, \bar{J})$. Say that $\delta$ and $\varepsilon$ are compatible if (1) $\delta$ induces $\varepsilon$ on $\bar{J}$ and (2) $\delta$ is an $\varepsilon$-homomorphism of the $J$ module $B$ (i. e., $\delta(x \cdot b)=\varepsilon(x) \cdot \delta(b)$ for $x \in J, b \in B$ ). Note that every $\alpha \in$ Aut $(K, \bar{K}, B)$ gives rise to a compatible pair $(\delta, \varepsilon)$ where $\delta$ is the restriction of $\alpha$ and $\varepsilon$ is induced by $\alpha$. Call $(K, \bar{K}, B)$ a weak triple of every compatible pair comes from some $\alpha \in$ Aut $(K, \bar{K}, B)$.

2. 3 Theorem. If $(K, \bar{K}, B)$ is a reeak triple then $(K, \bar{K}, B)$ is a $(t / u)$-triple for any compatible $(t, u)$.

Proof. Let $\phi(K) \in S^{\prime}$ where $\phi:(K, \bar{K}, B) \rightarrow\left(G, M, B_{0}\right)$ is an $E$-monomorphism. Let $\phi \mid \bar{K}=s$ and let $\phi$ induce $v: J \rightarrow J_{0}$. Define $\varepsilon=v^{-1} u: J \rightarrow J$. Since $u, v:(J, \bar{J}) \rightarrow\left(J_{0}, \bar{J}_{0}\right)$ we have $\varepsilon \in$ Aut $(J, \bar{J})$. Define $\delta: \bar{K} \rightarrow \bar{K}$ by $\delta=s^{-1} t$ so $\delta \in \operatorname{Aut}(\bar{K}, B)$. It is easily checked that $\delta$ and $\varepsilon$ are compatible. Let $\alpha \in \operatorname{Aut}(K, \bar{K}, B)$ with $\alpha \mid \bar{K}=\delta$ 
and $\alpha$ inducing $\varepsilon$. Define $\phi^{\prime}=\phi \alpha:(K, \bar{K}, B) \rightarrow\left(G, M, B_{0}\right)$. Then $\phi^{\prime} \mid \bar{K}=t$ and $\phi^{\prime}$ gives $u$ on $J$. Hence $\phi(K)=\phi^{\prime}(K) \in S$ proving the theorem.

Example. Let $\bar{J} \subset J \stackrel{\theta^{\theta}}{\rightarrow}$ Aut $B$ and $K=B \times{ }_{\theta} J, \bar{K}=B \times{ }_{\theta} \bar{J}$. If $\delta$ and $\varepsilon$ are compatible it follows that $\delta(\theta(x)(b))=\theta(\varepsilon(x)) \delta(b)$ for $b \in B$ $=B \times 1$ and $\delta x=\varepsilon x$ for $x \in \bar{J}=1 \times \bar{J}$. Set $\alpha=\delta \times \varepsilon$. Then $\alpha$ is an Aut $(K, \bar{K}, B)$ and gives $(\delta, \varepsilon)$. Hence $(K, \bar{K}, B)$ is a weak triple.

2. 4 Theorem. For each $J_{0} \in S_{1}=S(J \subset D)$ choose an isomorphism $u=u\left(J_{0}\right): J \rightarrow J_{0}$. Let $t: \bar{K} \rightarrow M$ be a fixed isomorphism. Suppose that $H^{2}\left(J, \bar{J}, u^{*} A\right)=0=H^{1}\left(J, \bar{J}, \operatorname{Hom}\left(B, u^{*} A\right)\right)$ for all choosen $u$. Suppose that $(K, \bar{K}, B)$ is a reak triple. Then

$$
S\left((K, \bar{K}) \subset G, E, B_{0}, M\right)=\cup_{J_{0} \in S_{1}} H^{1}\left(J, \bar{J}, u^{*} A\right) / H^{1}\left(J, \bar{J}, u^{*} t B\right) .
$$

Proof. Note first that $S\left((K, \bar{K}) \subset G, E, B_{0}, M\right)=\cup_{J_{0} \in S^{\prime}} S((K, \bar{K}) \subset$ $\left.G, E, B_{0} / J_{0}, M\right)$ and because $(K, \bar{K}, B)$ is a weak triple this last is $\underset{J_{0} \in S^{\prime}}{\subset} S(K \subset G, E, t / u)$. We will be finished if Corollary 1.5 can be applied; for this we must show that $S(K \subset G, E, t / u)$ is nonempty for $u: J \rightarrow J_{0}$. We have

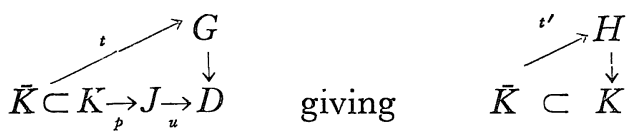

where $H=(u p)^{*} G$ the pull-back of $G$ along up. Thus a section of $H \rightarrow K$ extending $t^{\prime}$ will give the desired $\phi: K \rightarrow G$, making the first diagram commutative. Now define $o b\left(t^{\prime}\right)=\left[\mathscr{E}, t^{\prime}(K)\right] \in H^{2}(K, \bar{K}, A)$. Here $\mathscr{E}$ is the sequence $A \rightarrow H \rightarrow K$ and $H^{2}(K, \bar{K}, A)$ is identified with equivalence classes $[\mathscr{E}, L]$ by $[7$, Theorem 4.1]. I claim that $o b\left(t^{\prime}\right)=0$ iff $t^{\prime}$ has an extension to a section of $\mathscr{E}$. The reason is that by the proof in [7], $[\mathscr{E}, L]=0$ iff there is an isomorphism $\Gamma: E \rightarrow$ $K \times{ }_{\circ} A$ (semi-direct product) such that $\Pi \Gamma=p$ and $\Gamma(x)=(p(x), 1)=$ $i_{1}(x)$ for all $x \in L$. Now if $o b\left(t^{\prime}\right)=0$ then it is easy to see that $\Gamma^{-1} i_{1}$ is the desired extension. Conversely, if $s$ is such an extension then $\Gamma(e)=\left(p(e), e(s p(e))^{-1}\right)$ will be the required isomorphism. To 
complete the proof it will suffice to show that $H^{2}\left(K, \bar{K}, u^{*} A\right)=0$. There is an exact sequence

$$
0 \rightarrow H^{2}(J, \bar{J}, M) \rightarrow H^{2}(K, \bar{K}, M) \rightarrow H^{1}\left(J, \bar{J}, H^{1}(B, M)\right) \rightarrow \ldots
$$

(see [8]) and using $M=u^{*} A, H^{1}\left(B, u^{*} A\right)=\operatorname{Hom}\left(B, u^{*} A\right)$ and our hypotheses give $H^{2}(K, \bar{K}, A)=0$.

It may be worthwhile to discuss a relatively concrete example. Suppose $A \rightarrow G \rightarrow D$ is a given abelian extension. Let $S_{3}$ be the symmetric group on three letters. We ask for the copies of $S_{3}$ that are in $G$. First of all let $S_{1}=S\left(S_{3} \subset D\right)$ and $\alpha \in H^{2}(D, A)$ the classifying element of the given extension. For $J_{0} \in S_{1}$ let $\alpha \mid J_{0} \in$ $H^{2}\left(S_{3}, A\right)$ be the restriction of $\alpha$. Let $T=\left\{J_{0} \in S_{1}|\alpha| J_{0}=0\right\}$. If $S_{3} \cong L \subset G$ then we must have either $L \cap A=1$ or $L \cap A \cong C_{3}$. The $L$ 's with $L \cap A=1$ are the elements of $S\left(S_{3} \subset G, E, 1 / J_{0}\right)$ where $B \rightarrow K \rightarrow J$ is $1 \rightarrow S_{3} \rightarrow S_{3}$. Now $\left(S_{3}, 1,1\right)$ is a weak triple so that Theorem 2.3 applies. Corollary 1.5 gives $\cup_{J_{0} \in T} H^{1}\left(S_{3}, 1, u^{*} A\right)$ for these $L$ 's, where $u=u\left(J_{0}\right): S_{3} \rightarrow D$ is a chosen monomorphism.

Now we consider $L$ 's with $L \cap A=C_{3}$. These are the elements of $S\left(S_{3} \subset G, E, B_{0} / J_{0}\right)$ where $B \rightarrow K \rightarrow J$ is $C_{3} \rightarrow S_{3} \rightarrow C_{2}$. For each copy of $C_{2}$ in $D$ pick a monomorphism $v: C_{2} \rightarrow D$. For simplicity we make the following assumptions on the $C_{2}$-modules $v^{*} A$.

Assumption: $H^{2}\left(C_{2}, v^{*} A\right)=\operatorname{Hom}\left(C_{3}, v^{*} A\right) / \operatorname{Hom}_{C_{2}}\left(\left(C_{3}, v^{*} A\right)=\right.$ $H^{1}\left(C_{2}, \operatorname{Hom}\left(C_{3}, v^{*} A\right)\right)=0$.

The cohomology of cyclic groups is well known [see, e.g. 2] so these are easily checked in particular cases. From these assumptions we deduce easily that $H^{2}\left(C_{2}, 1, v^{*} A\right)=0=H^{1}\left(C_{2}, 1, \operatorname{Hom}\left(C_{3}, v^{*} A\right)\right)$ and Theorem 2.4 can be applied. $\left(S_{3}, C_{3}, C_{3}\right)$ is a weak triple because $S_{3}=C_{3} \times{ }_{\phi} C_{2}$. The final result is that

$$
\begin{aligned}
& S\left(S_{3} \subset G\right) \leftrightarrow {\left[\bigcup_{J_{0} \in T} H^{1}\left(S_{3}, 1, u^{*} A\right)\right] \cup } \\
& \bigcup_{(M, N) \in S_{2} \times S_{2}{ }^{\prime}} H^{1}\left(C_{2}, 1, v^{*} A\right) / H^{1}\left(C_{2}, 1, v^{*} s C_{3}\right)
\end{aligned}
$$

where $T$ is as above, $S_{2}=S\left(C_{2} \subset D\right), S_{2}{ }^{\prime}=S\left(C_{3} \subset A\right), v=v(M): C_{2} \rightarrow D$, $s=s(N): C_{3} \rightarrow A$ are chosen monomorphisms. If we know that two copies of $S_{3}$ in $D$ from $T$ are isomorphic by an automorphism of 
$D$ and similarly for any two copies of $C_{2}$ in $D$ then this simplifies to

$$
\begin{aligned}
S\left(S_{3} \subset G\right) & \leftrightarrow\left[T \times H^{1}\left(S_{3}, 1, A\right] \cup\left[S_{2} \times S_{2}^{\prime}\right.\right. \\
& \left.\times H^{1}\left(C_{2}, 1, A\right) / H^{1}\left(C_{2}, 1, C_{3}\right)\right]
\end{aligned}
$$

In Definition 2.2 we could drop the hypotheses $\bar{K} \supset B$. But then if $S=S\left((K, \bar{K}) \subset G, E, B_{0} / J_{0}, M\right) \ni L$ we have $L \triangleright B_{0}, L \supset M$ so $M^{1}=M B_{0}$ is a subgroup of $L$ and $S=S\left((K, \bar{K}) \subset G, E, B_{0} / J_{0}, M\right)$ with $\bar{K}=\bar{K} B, M=M B_{0}$.

\section{§ 3. Certain subgroups of solvable groups}

Let $G$ be a group (finite or infinite). Recall that a solvable series for $G$ is a descending chain

$$
G=G_{0} \supset G_{1} \supset \ldots \supset G_{s-1} \supset G_{s}=1
$$

with each $G_{i}$ normal in $G$ and each $G_{i-1} / G_{i}$ abelian. It will be convenient to write $G_{i}=F_{i} G$ and speak of the solvable series $F$ of $G$. The factors of the series are the groups $G_{i-1} / G_{i}=A_{i}(F)$, $i=1,2, \ldots, s$.

If $K$ is a subgroup of $G$ then it has induced filtration

$$
K=K_{0} \supset K_{1} \supset \ldots \supset K_{s-1} \supset K_{s}=1
$$

where $K_{i}=K \cap G_{i}$. $A_{i}$ gets a left $K / K_{i-1}$ module structure by $\left(x K_{i-1}\right)\left(y G_{i}\right)=\left(x y x^{-1}\right) G_{i}$ for $x \in K$ and $y \in G_{i-1}$. Let $B_{i}=B_{i}(K)=$ $K_{i-1} / K_{i} \subset A_{i}$. The $i$ 'th stage is then

$$
\begin{aligned}
& G_{i-1} / G_{i}=A_{i} \longrightarrow G / G_{i} \longrightarrow G / G_{i-1} \\
& \cup \quad \cup \quad \cup \\
& K_{i-1} / K_{i}=B_{i} \longrightarrow K / K_{i} \longrightarrow K / K_{i-1}
\end{aligned}
$$

Similarly each quotient group of $G$ has an induced filtration. When confusion is unlikely, all induced filtrations from $F$ will be denoted by $F$ also.

3. 1 Definition. (a) Suppose $K$ and $L$ are subgroups of $G$. A homomorphism $\phi: K \rightarrow L$ is an $F$-homomorphism if $\phi\left(K_{i}\right) \subset L_{i} i=1, \ldots$, s. It is an $F$-isomorphism if it has an inverse which is also an $F$-homomorphism. It is an $F$-monomorphism if it is an $F$-isomorphism 
onto its image.

(b) $S(K \subset G, F)=$ all subgroups of $G$ which are $F$-isomorphic to K.

(c) Let $K \supset \bar{K}$. Let $t: \bar{K} \rightarrow G, u_{i}: B_{i} \rightarrow A_{i}$ be given monomorphisms. Define $S\left(K \subset G, F,\left\{u_{i}\right\}, t\right)$ to be all $L \in S(K \subset G, F)$ such that there is an $F$-monomorphism $\phi: K \rightarrow G$ with $\phi_{i}=u_{i}: K_{i-1} / K_{i} \rightarrow$ $G_{i-1} / G_{i}$ and $\phi \mid \bar{K}=t$. If $\bar{K}=1$ drop it from the notation.

(d) Let $J_{i} \subset A_{i}=A_{i}(F), i=1,2, \ldots, s$, and $M \subset G$ be given subgroups. Define $S\left((K, \bar{K}) \subset G, F,\left\{J_{i}\right\}, M\right)$ to be all $L$ in $S(K \subset G, F)$ such that there is an $F$-monomorphism $\phi: K \rightarrow G, \phi\left(B_{i}\right)=J_{i}, \phi(\bar{K})=M$. Define $S((K, \bar{K}) \subset G, F, M)$ by requiring only $\phi(\bar{K})=M$.

(e) A subset of $S(K \subset G, F)$ is called a conjugate set if all its elements are conjugate. It is called a $K$-conjugate set if all its elements are conjugate to $K$.

For the next lemma we use the following notation.

$$
\begin{aligned}
& S=S\left((K, K) \subset G, F,\left\{J_{i}\right\}, i=1, \ldots, s, M\right) \\
& \left.S^{\prime}=S\left(\left(K / K_{s-1}\right), p \bar{K}\right) \subset G / G_{s-1}, F,\left\{J_{i}\right\}, i=1, \ldots, s-1, p M\right) \\
& S^{\prime \prime}(R)=S\left((K, \bar{K}) \subset G, E, J_{s} / R, M\right)
\end{aligned}
$$

Here $E$ is $1 \rightarrow G_{s-1} \rightarrow G \stackrel{p}{\rightarrow} G / G_{s-1} \rightarrow 1$. Let $\bar{J}_{s}=M \cap J_{s}$. Clarly we always have $S \subset \cup\left\{S^{\prime \prime}(R) / R \in S^{\prime}\right\}$. We are interested in finding sufficient conditions for equality.

3.2 Lemma. Let $J_{i}=B_{i}, M=\bar{K}$ normal in $G$. $B_{s}$ and $\bar{B}_{s}$ normal in $G$. Suppose $S^{\prime}$ is a $K / K_{s-1}$-conjugate set. Then $S^{\prime \prime}(R) \leftrightarrow S^{\prime \prime}\left(K / K_{s-1}\right)$ by conjugation. If $S^{\prime \prime}\left(K / K_{s-1}\right)$ is a conjugate set then so is each $S^{\prime \prime}(R)$ and $S$ is a K-conjugate set. Furthermore $S=\cup S^{\prime \prime}(R)$.

Proof. Let $c_{x}:\left(K / K_{s-1}, p \bar{K}\right) \rightarrow(R, p M)$ be an isomorphism where $c_{x}$ is conjugation by $x \in G / G_{s-1}$. Let $x=y G_{s-1}$, and consider $c_{y}$ : $S^{\prime \prime}\left(K / K_{s-1}\right) \rightarrow S^{\prime \prime}(R)$. Because the compositions of an $E$-isomorphism and $c_{y}$ is an $E$ isomorphism and because $B_{s}$ and $\bar{B}_{s}$ are normal we see that $c_{y}$ is well defined. The inverse is $c_{y}-1$ so $c_{y}$ gives the desired 1-1 correspondence. Now suppose $L \in S^{\prime \prime}(R)$ then $L$ is a conjugate 
of an element of $S^{\prime \prime}\left(K / K_{s-1}\right)$ which is in turn a conjugate of $K$. Thus $L$ is a conjugate of $K$ and hence in $S$ [The reason is that conjugation is an $F$-monomorphism. Specificaliy, if $G=G_{0} \supset G_{1} \supset \ldots$ $\supset G_{s}=1, G \triangleright G_{i}, K_{j}=K \cap G_{j}, y \in G, c_{y}: K \rightarrow G$ conjugation by $y$, then $c_{y}$ is an $F$-homomorphism and the equation $\left(c_{y} K\right)_{j}=c_{y}\left(K_{j}\right)$ shows that $c_{y-1}$ is also an $F$-homomorphism which will serve as an inverese to $c_{y}$.] Proving $S=\cup S^{\prime \prime}(R)$ and that $S$ is a conjugate set.

For the following theorem we suppose $G, F, K, \bar{K}$ given and take $J_{i}=B_{i}, M=\bar{K}$. Here $A_{i}=G_{i-1} / G_{i}, B_{i}=K_{i-1} / K_{i}, \bar{B}_{i}=\bar{K}_{i-1} / \bar{K}_{i}$. Suppose $\bar{K}$ normal in $G$ and each $B_{i}$ and $\bar{B}_{i}$ normal in $G / G_{i}$. Assume for $i=2, \ldots, s$.

Hyp. 1) $S\left(K / K_{i} \subset G / G_{i}, B_{i} /\left(K / K_{i-1}\right)\right)=\left\{K / K_{i}\right\}$ or Hyp. 2) $\left(K / K_{i}\right.$, $\left(\bar{K} K_{i} / K_{i}, B_{i}\right)$ a weak triple and $\operatorname{Ker} \delta: H^{1}\left(\bar{J}_{i}, B_{i}\right) \rightarrow H^{2}\left(J_{i}, \bar{J}_{i}, B_{i}\right)$ is 0 and $H^{1}\left(J_{i}, B_{i}\right) \rightarrow H^{1}\left(J_{i}, A_{i}\right)$ is isomorphic where $J_{i}=K / K_{i-1}, \bar{J}_{i}=$ $\bar{K} K_{i-1} / K_{i-1}$. Let $I=\{i \mid i=2, \ldots, s$, Hypothesis 2 is satisfied and Hypothesis 1 is not.

3. 3 Theorem. (1) $S=S\left(K \subset G, F,\left\{B_{i}\right\}, \bar{K}\right)$ is a conjugate set. (2) $S \leftrightarrow \prod_{i \in I} A_{i}^{J_{i}} /\left[A_{i}^{J_{i}}+B_{i}^{J_{i}}\right]$.

Proof. The proof is by induction on s. The result is trivally true for $s=1 \quad\left[S=\right.$ single element, $A_{1}^{J_{1}} / A_{1}^{J_{1}}+B_{1}^{J_{1}}=A / A+B=$ single element $]$ so we assume the result for filtrations of length $s-1$ and prove it for those of length $s$. We use the notation preceeding Lemma 3. 2 . Note that the induction assumption applies to both $S^{\prime}$ and $S^{\prime \prime}$. First suppose Hypothesis 1 is satisfied. Then $S^{\prime \prime}\left(K / K_{s-1}\right)$ is a conjugate set and Lemma 3. 2 shows $S=\cup S^{\prime \prime}(R)=S^{\prime} \times S^{\prime \prime}\left(K / K_{s-1}\right) \leftrightarrow S^{\prime}$ and $S$ is a conjugate set. This proves the theorem in the case. Now suppose Hypothesis 2 is satisfied. We have

$$
S(K \subset G, E, i d / i d) \rightarrow S^{\prime \prime}\left(K / K_{s-1}\right)
$$

and by our weak triple assumption and Theorem 2.3 these sets are equal. The hypotheses of Corollary 1.10 follow from those here so $S^{\prime \prime}\left(K / K_{s-1}\right)$ is a conjugate set. Lemma 3.2 now shows that $S$ is a 
conjugate set and $S \leftrightarrow S^{\prime} \times S^{\prime \prime}\left(K / K_{s-1}\right)$. Corollary 1.7 gives $S^{\prime \prime}\left(K / K_{s-1}\right)$ $\leftrightarrow A^{J} /\left[A^{J}+B^{J}\right]$ and completes the proof of the theorem.

The theorems until now have assumed $K \subset G$. I now want to include an existence statement. The resulting hypotheses, are, unfortunately, inductive in nature. However it will sometimes be possible to check them all at once rather than at each step.

Suppose $p: E \rightarrow D$ is a group homomorphism and $K$ a subgroup of $E$ and $B$ a subgroup of $D$. Say that $K$ covers $B$ if $p K=B$. Now suppose $G=G_{0} \supset G_{1} \supset \ldots \supset G_{s}=1$ is a solvable filtration of $G$ with $A_{i}=G_{i-1} / G_{i}$ and $A_{i} \supset B_{i}$. Say that a subgroup $K$ of $G$ covers $B_{i}$ provided $K_{i-1}$ covers $B_{i}$.

Let $B_{i}$ be normal in $G / G_{i}$. If $G / G_{i-1} \supset W$ and $W$ covers $B_{1}, \ldots B_{i-1}$ then assume $H^{2}\left(W, A_{i} / B_{i}\right)=0$ for this $W$ and also

either Hypothesis. 1) there can be at most one subgroup $G / G_{i}$ covering $B_{i}$ and $W$

or Hypothesis. 2) if $W^{\prime}$ covers $W$ and $B_{i}$ then $\left(W^{\prime}, B_{i}\right)$ is a weak pair and $H^{1}\left(W, B_{i}\right) \rightarrow H^{1}\left(W, A_{i}\right)$ is isomorphic.

3. 4 Theorem. In the above sitting

(1) There is a subgroup $K$ of $G$ covering $B_{1}, \ldots, B_{s}$.

(2) $S=S\left(K \subset G, F,\left\{B_{i}\right\}\right)$ is a conjugate set.

(3) Let $I=\{i \mid i=2, \ldots$, s such that Hypothesis 2 is satisfied and Hypothesis 1 is not $\}$. Then $S \leftrightarrow \prod_{i \in I} A_{i} /\left[A_{i}^{K / K_{i-1}}+B_{i}\right]$.

Proof. We may assume the result for $G / G_{s-1}$ so we have $W_{s-1}$ covering $B_{1}, \ldots, B_{s-1}$. Consider the following diagram

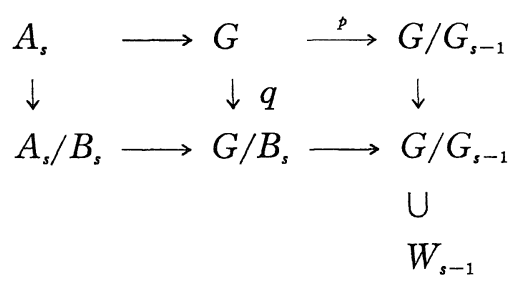

The middle extension splits when pulled back over $W_{s-1}$ and there results a monomorphism of $W_{s-1}$ into $G / B_{s}$ with image, say, $W_{s}^{\prime}$. 
$q^{-1} W_{s}^{\prime}$ covers $W_{s-1}$ and $B_{s}$. This proves (1) of the theorem. Parts (2) and (3) are now consequences of Theorem 3.3 with $\bar{K}=1$.

Theorem 3.4 and the corollaries that follow can be viewed as generalizations of P. Hall's theoerm [1]. The reader may wish to compare them to some other generalizations from a somewhat different viewpoint $[5,6]$.

3. 5 Corollary. In 3.4 (2) can be replaced by (2') any two subgroups covering $B_{1}, \ldots, B_{s}$ are conjugate.

Proof. In the proof of 3.4 we assume (2) and $\left(2^{\prime}\right)$ at the $(s-1)-$ stage. Suppose $\bar{W}$ covers $B_{1}, \ldots, B_{s}$ and consider the diagram at the beginning of the proof of 3.4. Let $\bar{W}^{\prime}=q \bar{W} . \quad p^{\prime} \bar{W}^{\prime}$ covers $B_{1}, \ldots, B_{s-1}$ so it is in $S^{\prime} . \quad p^{\prime}$ is an isomorphism on $\bar{W}^{\prime}$ and $W^{\prime}$ so $\bar{W}^{\prime}$ is $F$-isomorphic to $W^{\prime}$ and so conjugate to it (by the theorem). Now the conjugation lifts to show $\bar{W}$ and $W$ are conjugate.

Consider

$H^{2}\left(G / G_{i-1}, A_{s}\right) \stackrel{q^{*}}{\longrightarrow} H^{2}\left(G / G_{i-1}, A_{i} / B_{i}\right) \stackrel{\text { rest }}{\longrightarrow} H^{2}\left(W, A_{i} / B_{i}\right)$ and let $E_{i}=\left(A_{i} \rightarrow G \rightarrow G / G_{i-1}\right) \in H^{2}\left(G / G_{i-1}, A_{i}\right)$. Then the proof shows the following corollary to be true.

3. 6 Corollary. In 3.4 the hypothesis $H^{2}\left(W, A_{i} / B_{i}\right)=0$ can be replaced by rest $q_{*}\left(E_{i}\right)=0$.

Recall the following classical theorem of P. Hall [1]. Let $G$ be a solvable group of order $m n$ with $(m, n)=1$. Call $H$ a Hall subgroup of $G$ if $|H|=m$. Then any two Hall subgroups are conjugate. Let $h$ be the number of Hall subgroups of $G$. Then $h$ is the product of factors $f_{i}$ such that (a) $f_{i} \equiv 1\left(\bmod p_{i}\right)$ and $p_{i}$ is a prime factor of $m$, and (b) $f_{i}$ is a power of a prime and divides one of the chief factors of $G$. Now form a chief series for $G, G=G_{0} \supset G_{1} \supset \ldots \supset G_{s}=1$. The $A_{i}$ are elementary abelian. If $\left|A_{i}\right|=q^{f}$ then $q \mid m$ or $q \mid n$. If $q \mid m$ set $B_{i}=A_{i}$ and if $q \mid n$ set $B_{i}=1$. If $W$ covers $B_{i}, \ldots, B_{s}$ then $|W| \mid m$ and is relatively prime to $\left|A_{i} / B_{i}\right|$ and so $H^{2}\left(W, A_{i} / B_{i}\right)=0$. 
If $B_{i}=A_{i}$ then Hypothesis 1 is satisfied. If $B_{i}=1$ then $\left(W^{\prime}, B_{i}\right)$ is a weak triple, $H^{1}\left(W, B_{i}\right)=0=H^{1}\left(W, A_{i}\right)$ since $|W|$ and $\left|A_{i}\right|$ are relatively prime. Corollary 3.5 gives the conjugacy of the Hall subgroups and that $h=\left|\prod_{i \in I} A_{i} / A_{i}^{K / K_{i-1}}\right|$ where $I$ is the set of $i$ with $B_{i}=1$. Part (b) is clear and part (a) follows from induction and the following elementary counting fact: If $A$ is an elementary $q$ group of order $q^{n}$ acted on by a $p$ group $P$ then $\left|A^{P}\right|=q^{f} \Rightarrow q^{n-f} \equiv 1$ $(\bmod p)$.

The above discussion includes a proof of the following.

3. 7 Corollary. Suppose $G$ is a group and $G=G_{0} \supset G_{1} \supset \ldots \supset G_{s}=1$ a solvable series with $A_{i}=G_{i-1} / G_{i}$. Let $\{2, \ldots, s\} \supset I$ and $B_{i}=1, i \in I$, $B_{i}=A_{i} \quad i \notin I$.

Suppose that if $i \in I$ and $G / G_{i-1} \supset W$ and $W$ covers $B_{1}, \ldots, B_{i-1}$ then $H^{j}\left(W, A_{i}\right)=0, j=1,2$.

Then (1) $G$ has a subgroup $K$ covering $B_{1}, \ldots, B_{s}$ and any two such are conjugate (2) If $S=$ all subgroups covering $B_{1}, \ldots, B_{s}$ then $S \leftrightarrow \prod_{i \in I} A_{i} / A_{i}^{\left(K / K_{i-1}\right)}$.

\section{Appendix}

I will recall here a few facts about relative cohomology from [Takasu, 3] for the readers benefit. I will also prove the equivalence of the first cohomology group and the derivation group since this was not done explicitly by Takasu.

Let $G$ be a group, $H$ a subgroup, and $A$ a $G$-module. $I(G, H, A)$ is the kernel of the natural epimorphism $Z(G) \otimes_{H} A \rightarrow A, g \otimes a \rightarrow g a$, and $J(G, H, A)$ is the cokernel of the natural monomorphism $A \rightarrow \operatorname{Hom}_{H}(Z(G), A), a \rightarrow(g \rightarrow g a) . \quad H^{q}(G, H, A)$ is defined to be $\operatorname{Ext}^{q-1}(I(G, H, Z), A)$ for $q \geqq 1$. There is a long exact sequence for the pair $(G, H)$,

$$
\cdots \rightarrow H^{q}(G, H, A) \rightarrow H^{q}(G, A) \rightarrow H^{q}(H, A) \rightarrow H^{q+1}(H, A) \rightarrow \cdots
$$

and also a long exact sequence for $0 \rightarrow A^{\prime} \rightarrow A \rightarrow A^{\prime \prime} \rightarrow 0$ (a short exact sequence of $G$-modules) 


$$
\begin{aligned}
& \cdots \rightarrow H^{q}\left(G, H, A^{\prime}\right) \rightarrow H^{q}(G, H, A) \rightarrow \\
& H^{q}\left(G, H, A^{\prime \prime}\right) \rightarrow H^{q+1}\left(G, H, A^{\prime}\right) \rightarrow \cdots
\end{aligned}
$$

Relative cohomology can be reduced to absolute cohomology by

$$
H^{q}(G, H, A)=H^{q-1}(G, J(G, H, A)) \text {. }
$$

Let $G \supset H \supset N$ and $G \triangleright N$. There is a spectral sequence which gives in particular

$$
H^{1}\left(G / N, H / N, A^{N}\right) \approx H^{1}(G, H, A)
$$

and an exact sequence

$$
\begin{aligned}
0 \rightarrow & H^{2}\left(G / N, H / N, A^{N}\right) \rightarrow H^{2}(G, H, A) \rightarrow \\
& H^{1}\left(G / N, H / N, H^{1}(N, A)\right) \rightarrow H^{3}\left(G / N, H / N, A^{N}\right) \rightarrow \\
& H^{3}(G, H, A) .
\end{aligned}
$$

Now write $I(G, H, Z)=I(G, H)$. The definition tells us that $H^{1}(G, H, A)=\operatorname{Hom}_{G}(I(G, H), A)$. Also we have (by definition)

$$
\begin{aligned}
& 0 \rightarrow I(G, H) \rightarrow Z(G) \otimes_{H} Z \stackrel{\dot{s}}{\rightarrow} Z \rightarrow 0 \\
& 0 \rightarrow I(G) \rightarrow Z(G) \stackrel{\mathfrak{0}_{0}}{\rightarrow} Z \rightarrow 0
\end{aligned}
$$

where $\varepsilon_{0}\left(\sum n_{i} x_{i}\right)=\sum n_{i}$ and $\varepsilon(r \otimes m)=\varepsilon_{0}(r) m$. Define

$$
\begin{aligned}
\operatorname{Hom}^{\prime}\left(Z G \otimes_{H} Z, A\right)=\{g & \in \operatorname{Hom}\left(Z G \otimes_{H} Z, A\right) \mid g(\mathrm{rs} \otimes 1) \\
& \left.\left.=r g(s \otimes 1)+g(r \otimes 1) \varepsilon_{0}(s)\right)\right\}
\end{aligned}
$$

and check that it is a subgroup.

Theorem. $\operatorname{Der}(G, H, A) \cong \operatorname{Hom}^{\prime}\left(Z G \otimes_{H} Z, A\right) \cong \operatorname{Hom}_{G}(I(G, H), A)=$ $H^{1}(G, H, A)$

Proof. [cf. MacLane, 4, pp. 106-107]. Let $g \in \operatorname{Der}(G, H, A)$ and define $g^{\prime} \in \mathrm{Hom}^{\prime}$ by $\mathrm{g}^{\prime}(x \otimes 1)=g(x)$. It is a well defined homomorphism since $Z G \otimes_{H} Z$ is free abelian on $G \backslash H$. It is easily checked to be in Hom'. For $u \in$ Hom define $\hat{u}$ in $\operatorname{Hom}_{G}(I(G, H), A)$ by $\hat{u}=u \mid I(G, H)$ and check that it is a well defined $G$-homomorphism.

Now define $p: Z G \bigotimes_{H} Z \rightarrow I(G, H)$ by $p(r \otimes 1)=r \otimes 1-\varepsilon(r)(1 \otimes 1)$. If $h \in \mathrm{Hom}_{G}$ then $h \rightarrow h p$ is a homomorphism to Hom'. $v \rightarrow v \mid G$ is a homomorphism Hom' $\rightarrow$ Der. It is easy to check that these maps 
give the desired isomorphisms.

The lemma reveals the relationship between the cohomology of Barr-Rinehart [1,2] and that of Takasu [8]. It also suggests that Barr-Rinehart made an unfortunate (although defensible) choice of indexing. Recall that Barr-Rinehart defined $\bar{H}_{\phi}^{n}(G, A)$ via derivations where $\phi: G \rightarrow D$ is a group homomorphism. They noted that for $\phi=$ identity, $\bar{H}_{\phi}^{n}(G, A)=H^{n+1}(G, A), n \geqq 1$, where the last is group cohomology. Now let $I_{\phi}(G, H)=I(G, H) \otimes_{z G} Z D$ and define $H_{\phi}^{q}(G, H, A)=$ $\operatorname{Ext}_{G}^{q-1}\left(I_{\phi}(G, H), A\right)$ for $q \geqq 1$ [cf. 2, p. 207]. If $\phi=i d$. then this is group cohomology. It is easy to see that $\bar{H}_{\phi}^{n}(G, A)=H_{\phi}^{n+1}(G, 1, A)$, $n \geqq 0$, and in particular $\bar{H}^{n}(G, A)=H^{n+1}(G, 1, A), n \geqq 0$. The $H$ indexing and relative notation seem best to me since they are consistent with topology and should make the relationship between results in algebraic and topological cohomology transparent.

\section{References}

[1] Barr, M., and Rinehart, G. S., Cohomology as the derived functor of derivations, Trans. A mer. Math. Soc., 122 (1966), 416-426.

[2] Eckmann, B., and Stammbach, U., On exact sequences in the homology of groups and algebras, Ill. J. Math., 14 (1970), 205-215.

[3] Hall, Phillip, A note on soluble groups, J. London Math. Soc., 3 (1928), 98-105.

[4] Maclane, S., Ho mology, Springer-Verlag, New York, 1963.

[5] Mann, A., System normalizers and subnormalizers, Proc. London Math. Soc., (3) 20 (1970), 123-143.

[6] Prentice, M. J., X-normalizers and X-covering subgroups, Proc. Camb. Phil. Soc., 66 (1969), 215-230.

[7] Takasu, S., On the cha' ge of rings in the homological algebra, J. Math. Soc. Japan, 9 (1957), 315-329.

[8] Takasu, S., Relative homology and relative cohomology theory of groups, J. Fac. Sci. Univ. Tokyo, Sect. I, 8 (1959-60), 75-110. 